\title{
High-Field Pauli-limiting behavior and Strongly Enhanced Upper Critical Magnetic Fields near the Transition Temperature of an Arsenic-Deficient $\mathrm{LaO}_{0.9} \mathrm{~F}_{0.1} \mathrm{FeAs}_{1-\delta}$ Superconductor
}

\author{
G. Fuchs, S.-L. Drechsler*, N. Kozlova, G. Behr, A. Köhler, J. Werner, K. Nenkov, R. Klingeler, J. Hamann-Borrero, \\ C. Hess, A. Kondrat, M. Grobosch, A. Narduzzo, M. Knupfer, J. Freudenberger, B. Büchner, and L. Schultz \\ IFW Dresden, P.O. Box 270116, D-01171 Dresden, Germany
}

(Dated: October 24, 2018)

\begin{abstract}
We report upper critical field $B_{c 2}(T)$ data for disordered (arsenic-deficient) $\mathrm{LaO}_{0.9} \mathrm{~F}_{0.1} \mathrm{FeAs}_{1-\delta}$ in a wide temperature and magnetic field range up to $47 \mathrm{~T}$. Because of the large linear slope of $B_{c 2} \approx-5.4$ to $-6.6 \mathrm{~T} / \mathrm{K}$ near $T_{c} \approx 28.5 \mathrm{~K}$ the $T$-dependence of the in-plane $B_{c 2}(T)$ shows a flattening near $23 \mathrm{~K}$ above $30 \mathrm{~T}$ which points to Pauli-limited behavior with $B_{c 2}(0) \approx 63$ to $68 \mathrm{~T}$. Our results are discussed in terms of disorder effects within conventional and unconventional superconducting pairings.
\end{abstract}

The recent discovery of relatively high transition temperatures $T_{c}$ in $\mathrm{LaO}_{1-x} \mathrm{~F}_{x} \mathrm{FeAs}$ [1] has established a new family of superconductors. Since the usual el-ph mechanism has been ruled out by a much too weak coupling strength $\lambda \leq 0.2[2]$, a variety of nonstandard mechanisms mostly involving spin fluctuations has been proposed [3, 4, 5]. Naturally, our knowledge about these fascinating systems is still poor at present. Together with the controversially discussed unconventional ( $p$ - or $d$ wave) vs conventional (extended $s$-wave) symmetry and magnitude of the superconducting order parameter $\Delta$ (gap) [6, 7], the upper critical field $B_{c 2}(T)$ and its slope near $T_{c}$ are fundamental quantities characterizing the superconducting state. Due to the involved Fermi velocities $v_{f}$ in the clean limit it provides insight into the underlying electronic structure which might be affected by a complex interplay of correlation effects [8, 9] and the vicinity of competing magnetism [10, 11, 12]. Controlled disorder provides insight into relevant scattering processes and in the symmetry of the pairing since any unconventional pairing in the sense of $T_{c}$ and $d B_{c 2} /\left.d T\right|_{T_{c}}$ is expected to be suppressed by strong disorder [13, 14, 15, 16]:

$$
-\ln \left(\frac{T_{c}}{T_{c 0}}\right)=\psi\left(\frac{1}{2}+\frac{\beta T_{c 0}}{2 \pi T_{c}}\right)-\psi\left(\frac{1}{2}\right)
$$

where $\psi(x)$ is the digamma function and $\beta$ is the strongcoupling pair-breaking parameter $\beta=\Omega_{p}^{2} \rho_{0} / 8 \pi(1+\lambda) T_{c 0}$ which is related to the residual resistivity $\rho_{0}$ and the plasma energy $\Omega_{p}$ in the $(a, b)$-plane. Some, but much weaker, suppression might occur also in the anisotropic or multiband $s$-wave case since the scattering may smear out the gap anisotropy. However, it will be shown that surprisingly nothing similar happens in our case.

For low applied fields rather different slopes $d B_{c 2} / d T \approx-1.6 \mathrm{~T} / \mathrm{K}$ up to $-2 \mathrm{~T} / \mathrm{K}$ at $T_{c} \approx 26 \mathrm{~K}[17,18]$ and up to $-4 \mathrm{~T} / \mathrm{K}$ at $T_{c} \approx 20 \mathrm{~K}$ [19] have been reported for the As stoichiometric La based compounds. Here, we report with $d B_{c 2} / d T \approx-5.4$ to $-6.6 \mathrm{~T} / \mathrm{K}$, to our knowledge one of the highest slopes of $B_{c 2}$ near $T_{c}$ observed so far for the La-series. Another interesting is- sue of high-field studies considered here is the possibility of Pauli-limiting (PL) behavior. Triplet $p$-wave pairing or strong coupling $\left(B_{c 2}(0) \leq 50 \mathrm{~T}\right)$ would naturally explain the reported absence of PL [17]. In this context it is important that we succeeded to detect PL behaviour for our specific sample. It points to $B_{c 2}(0)$ values being much below often-used WHH (Werthamer-HelfandHohenberg) [20] based estimates . After presenting various data which deviate from those of Ref. 17 as well as from our non-deficient As samples [21, 22, 23, 24, 25], we will discuss our $B_{c 2}(T)$ results in the light of these more general issues.

A polycrystalline sample of $\mathrm{LaO}_{0.9} \mathrm{~F}_{0.1} \mathrm{FeAs}_{1-\delta}$ was prepared as described in our previous work 21, 22, 23, 24, 25] and e.g. in Ref. 26. However, in contrast to that work here a Ta foil was used to wrap the pellets. Ta acts as an As getter at high $T$ forming a solid solution of about 9.5 at\% As in Ta with a small layer of $\mathrm{Ta}_{2} \mathrm{As}$ and TaAs on top of the foil. This leads to an As loss in the pellets. The annealed pellets were ground and polished and the local composition of the resulting samples was investigated by wavelength-dispersive x-ray spectroscopy (WDX) in a scanning electron microscope. The sample consists of 1 to $20 \mu \mathrm{m}$ sized grains of $\mathrm{LaO}_{0.9} \mathrm{~F}_{0.1} \mathrm{FeAs}_{1-\delta}$ $(\delta \sim 0.05$ to 0.1$)$ where the $\mathrm{F}$ content slightly fluctuates between different grains. A powder x-ray diffraction study with a Rietveld refinement of the main phase yields slightly enhanced lattice constants: $a=4.02819 \AA$ and $c=8.72397 \AA$ compared to $a=4.02043(4.02451) \AA$ and $c=8.69552(8.70995) \AA$ for our cleanest sample with $T_{c}=26.8 \mathrm{~K}$ and an ordinary sample with $T_{c}=26 \mathrm{~K}$, respectively [25]. The latter are almost As-stoichiometric samples with the same $\mathrm{F}_{0.1}$-content. Surprisingly, the lattice constants of the ADS (As deficient sample) are close to those for underdoped samples near the border of magnetism and superconductivity in stoichiometric samples. The reduced charge of the anionic As layers suggests less attraction between them and the adjacent "cationic" central $\mathrm{Fe}$ and the charge reservoir $\mathrm{LaO}_{0.9} \mathrm{~F}_{0.1}$ layers. This might explain the increase of $c$ and due to the weakened 


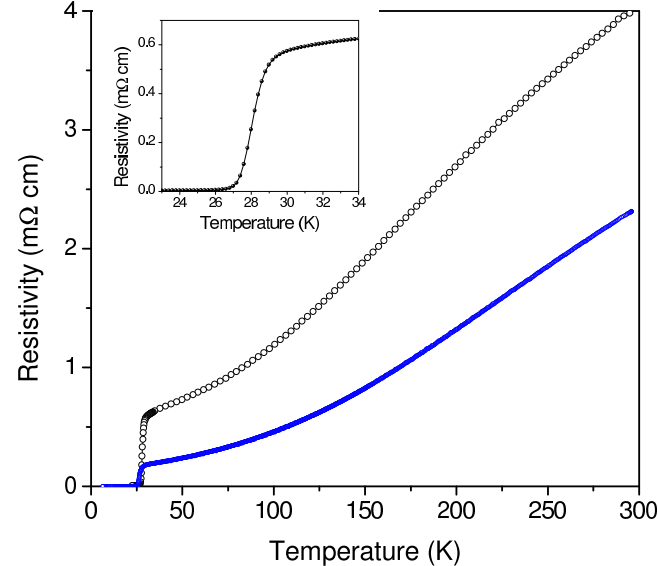

FIG. 1: (Color online) Resistivity at zero magnetic field of the $\mathrm{LaO}_{0.9} \mathrm{~F}_{0.1} \mathrm{FeAs}_{1-\delta}, \delta \approx 0.05$ to $0.1 \mathrm{ADS}$ studied in the present paper. The inset shows the resistivity near $T_{c}$. Solid line: a cleaner almost stoichiometric sample 25].

Madelung potential also a reduced doping of the Fe-layer.

The electrical resistance was measured for a platelike $\mathrm{LaO}_{0.9} \mathrm{~F}_{0.1} \mathrm{FeAs}_{1-\delta}$ ADS with nominal dimensions $3 \times 2.6 \times 0.53 \mathrm{~mm}^{3}$ using the standard four-point method. Its resistivity $\rho(T)$ is shown in Fig. 1. The resistivity of this ADS in the normal state at $30 \mathrm{~K}$, with about $0.6 \mathrm{~m} \Omega \mathrm{cm}$, clearly exceeds that reported for other $\mathrm{LaO}_{0.9} \mathrm{~F}_{0.1} \mathrm{FeAs}$ samples. Nevertheless, our ADS was found to exhibit a rather high $T_{c}$-value of $28.5 \mathrm{~K}$ defined at $90 \%$ of $\rho$ in the normal state and a relatively sharp transition width (see inset of Fig. 1) which excludes an anomalous inhomogeneity. The low- $T$ region above $T_{c}$ with $\rho \propto T^{2}$, ascribed to a pronounced el-el scattering was regarded as evidence for a standard Fermi liquid picture [18], has been somewhat narrowed from $225 \mathrm{~K}$ to $T \leq 175 \mathrm{~K}$ for this ADS 27]. The $\rho(T)$-dependence of this ADS resembles that of underdoped stoichiometric

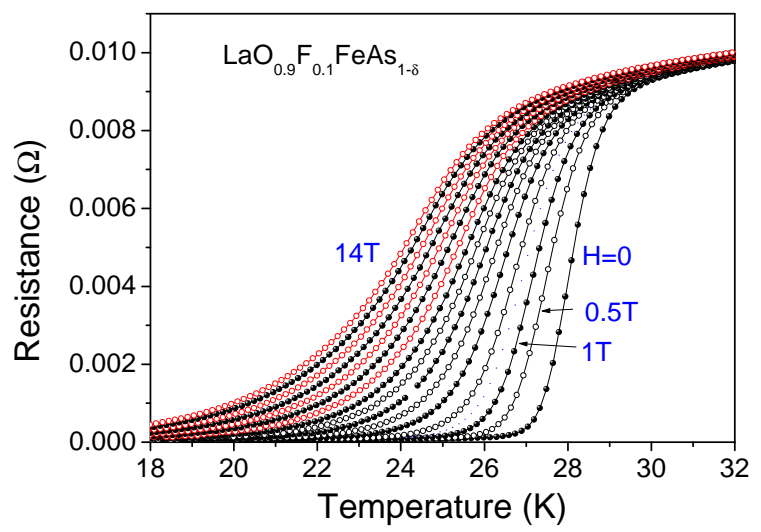

FIG. 2: (Color online) $T$-dependence of the resistance $R$ for the ADS for various dc fields up to $14 \mathrm{~T}$. Between 1 and $14 \mathrm{~T}$, the applied magnetic field was increased in steps of $1 \mathrm{~T}$.

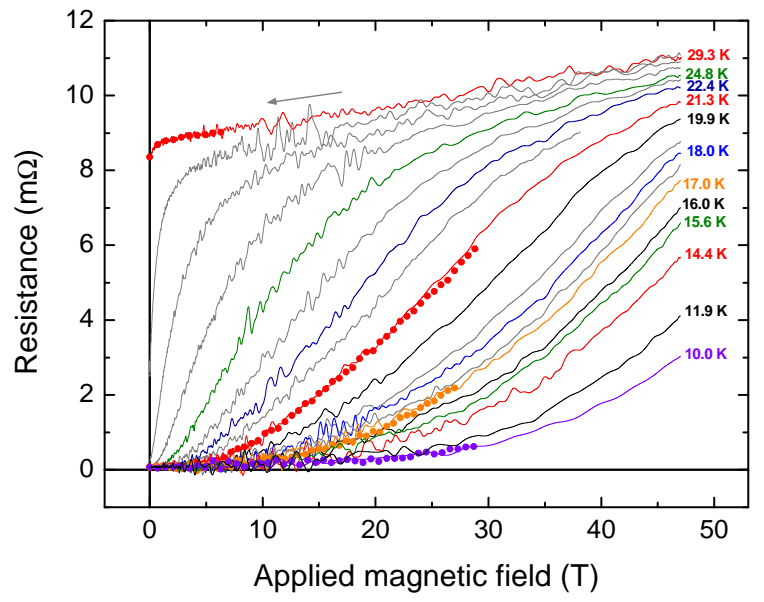

FIG. 3: (Color online) Field dependence of the resistance at fixed $T$ (see legend) measured in pulsed fields. Lines: measurements up to $47 \mathrm{~T}$; symbols: measurements shown for four selected $T$-values.

samples in the range of 0.05 to $0.07 \mathrm{~F}$-content 25]. Since each As site is surrounded by four Fe sites, the effect of even few As vacancies might be drastic. Thus, a substantial shortening of the mean free path $l$ up to few lattice constants $a$ as estimated below from the observed enhanced field slope at $T_{c}$ seems to be quite reasonable.

In Fig. 2, the electrical resistance of the studied ADS is plotted vs. $T$ for applied dc fields up to $14 \mathrm{~T}$. Resistance data vs. applied field of this ADS measured in pulsed fields up to $50 \mathrm{~T}$ are plotted in Fig. 3. Gold contacts (100 nm thick) were made by sputtering in order to provide a low contact resistivity and therefore to avoid possible heating effects in the high-field measurements performed in the $50 \mathrm{~T}$ magnet of the Dresden-HighMagnetic-Field-Laboratory [28]. The agreement between measurements up to $29 \mathrm{~T}$ and $47 \mathrm{~T}$ confirms that our data are not affected by sample heating. At high fields a substantial broadening of the transition curves is observed as shown in both figures. It stems from the large anisotropy of $B_{c 2}(T)$ expected for layered compounds as here 4, 12]. $B_{c 2}$ was determined as in Ref. 17 from the onset of superconductivity defining it at $90 \%$ of $R_{N}$, the resistance in the normal state. Within a second approach the magnetoresistance in the normal state was taken into account and $R_{N}(T)$ has been described as explained in Ref. 27 . For this modified definition of $B_{c 2}$ one gets somewhat higher $B_{c 2}$-values and also higher slopes near $T_{c}$.

Generally, the $B_{c 2}$ values from $0.9 R_{N}$ data refer to those grains which are oriented with their $a b$-planes along the applied field. The $B_{c 2}^{a b}(T)$ curve of our ADS is shown in Fig. 4. The comparison of the data from dc and pulsed field measurements in the field range up to $14 \mathrm{~T}$ confirms that both data sets do well agree. The $B_{c 2}(T)$-curve in Fig. 4 shows a surprisingly steep $d B_{c 2} /\left.d T\right|_{T_{c}}=-5.4 \mathrm{~T} / \mathrm{K}$ (-6.6 $\mathrm{T} / \mathrm{K}$ within the second approach described above) 
which exceeds the slopes reported for cleaner non-ADS samples by more than a factor of two [17, 18, 26]. This points to strong impurity scattering in our ADS in accord with its enhanced resistivity at $30 \mathrm{~K}$. For applied fields up to about $30 \mathrm{~T}$, the $B_{c 2}(T)$ data can be well described by the standard WHH model [20]. Using $d B_{c 2} / d T=-5.4$ $\mathrm{T} / \mathrm{K}(-6.6 \mathrm{~T} / \mathrm{K})$ and $T_{c}=28.5 \mathrm{~K}(28.8 \mathrm{~K})$, this model predicts $B_{c 2}^{*}(0)=-0.69 T_{c}\left(d B_{c 2} / d T\right)_{T_{c}}=106 \mathrm{~T}(131 \mathrm{~T})$ at $T=0$. However, for applied fields above $30 \mathrm{~T}$ or below $23 \mathrm{~K}$ increasing deviations of the $B_{c 2}(T)$ data from the WHH-curve are clearly visible. They increase with applied field. Notice that the resulting difference between the measured $B_{c 2}(T)$ and $B_{c 2}^{*}$ is comparable for both definitions of the upper critical field (Fig. 5). The flattening of $B_{c 2}(T)$ at high fields points to its limitation by the Pauli spin paramagnetism. This effect is measured in the WHH model by the Maki parameter $\alpha$

$$
\alpha=\sqrt{2} B_{c 2}^{*}(0) / B_{p}(0),
$$

where $B_{p}(0)$ is the paramagnetically limited field [29]:

$$
B_{p}(0)[\text { Tesla }]=1.86 \eta_{\text {eff }}(\lambda) T_{c}[\mathrm{~K}],
$$

where $\eta_{\text {eff }}=(1+\lambda)^{\varepsilon} \eta_{\Delta} \eta_{B_{c 2}}(1-I)$ is a correction to BCS due to the e-boson and e-e interaction $(I=N(0) V$ is the Stoner factor) [29, 30]. $\eta_{\text {eff }}$ depends on the elboson coupling constant $\lambda$ and $\varepsilon=0.5,1$ according to Refs. 29, 30, respectively. Due to PL, $B_{c 2}(0)$ is lowered:

$$
B_{c 2}^{p}(0)=B_{c 2}^{*}(0) / \sqrt{1+\alpha^{2}} .
$$

Ignoring weak spin-orbit scattering for the sake of simplicity, we get $\alpha=1.31$ and $B_{c 2}^{p}(0)=63 \mathrm{~T}(68 \mathrm{~T})$ from Eqs. (2)-(4), using $\lambda=0.5$ [23] for a representative value of the el-boson coupling constant for $\mathrm{LaO}_{0.9} \mathrm{~F}_{0.1} \mathrm{FeAs}_{1-\delta}$, $\eta_{\text {eff }}=2.09$, and $B_{c 2}^{*}(0)=106 \mathrm{~T}(131 \mathrm{~T})$ without PL. The $B_{c 2}^{p}(T)$ line plotted in Fig. 4 is based on Eq. (4) and was obtained by replacing $B_{c 2}^{*}(0)$ entering its numerator and $\alpha$ by $B_{c 2}^{*}(T, \alpha=0)$ of the WHH model. This rough approximation of $B_{c 2}^{p}(T)$ has been used to illustrate the $\mathrm{PL}$ in the studied ADS.

High $B_{c 2}(0)$-values and similarly for $d B_{c 2} /\left.d T\right|_{T_{c}}$ can be achieved by: (i) strong coupling, (ii) small Fermi velocities $v_{f}$, and (iii) strong intra-band scattering. Strong coupling can be excluded empirically for related systems [6, 23]. Note that unconventional triplet $p$ - or $d$-wave pairing [13, 14, 15] and (iii) can not be reconciled. Hence, singlet extended $s$-wave pairing remains as a reasonable scenario. As mentioned above the residual resistivity $\rho_{0}$ of our ADS is enhanced compared to that of clean samples (Fig. 1) examined so far in our studies. This points to strong intraband scattering. Adopting in accord with the single isotropic gap found for $\mathrm{SmFeAsO}_{0.85} \mathrm{~F}_{0.15}$ [6] an effective single-band $s$-wave picture 31], $B_{c 2}^{c}(0)$ (and similarly for $\left.B_{c 2}^{a b}(0)\right)$, written in convenient units, ignoring $\mathrm{PL}$ reads in the clean $(\mathrm{cl})$ and general case:

$$
B_{c 2, c l}^{c}(0)[\mathrm{T}]=0.0237(1+\lambda)^{2.2} T_{c}^{2}[\mathrm{~K}] / v_{f}^{2}\left[10^{5} \mathrm{~m} / \mathrm{s}\right]
$$

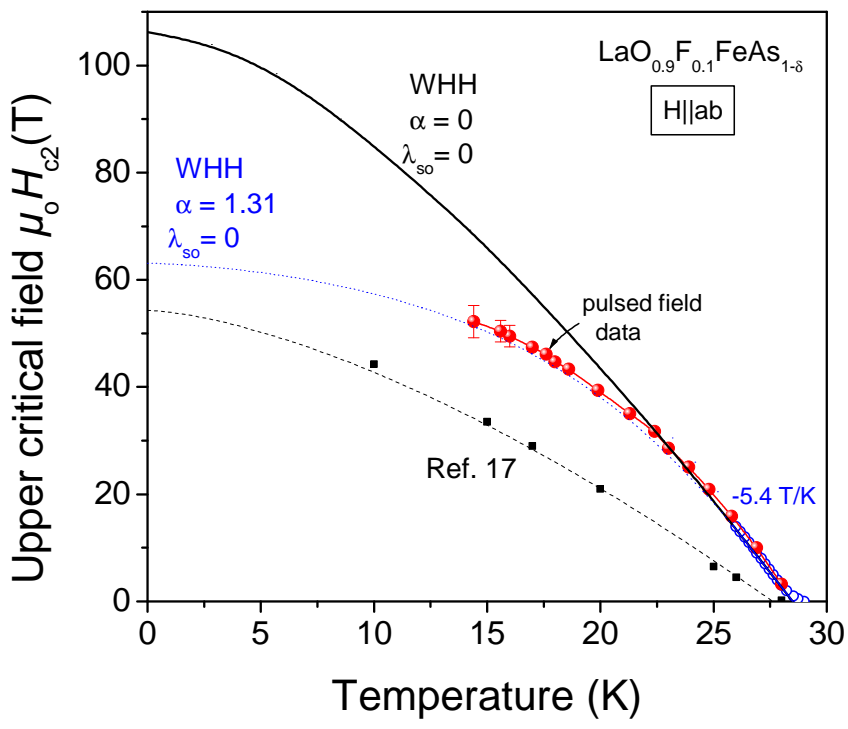

FIG. 4: $B_{c 2}^{a b}$ vs. T. Data from dc (०) and pulsed field measurements $(\bullet)$. The last three "data points" above $47 \mathrm{~T}$ are obtained from Fig. 3 by a linear extrapolation of $R(B)<$ $0.9 R_{N}$ to $R(B)=0.9 R_{N}$. Dashed line: data from Ref. 17; solid line: WHH-model without PL. Dotted line: $B_{c 2}^{*}(T)$ for $\alpha=1.31$ without spin-orbit scattering.

$$
B_{c 2}^{c}(0)=B_{c 2, c l}(0)\left[1+0.13 \gamma_{i m p}[\mathrm{~K}] /\left(T_{c}(1+\lambda)\right)\right],
$$

where $\lambda$ is the relevant e-boson coupling constant, $\gamma_{i m p}$ is the scattering rate, and $v_{f}$ denotes the in-plane averaged Fermi velocity. The mass anisotropy $\gamma^{2}=$ $M / m$ which affects $B_{c 2}^{a b}$ varies according to the LDApredictions [4, 12, 23] between 6.2 and 15. From the $5 \%$ level of the resistivity transition curves (see Fig. 5) a lower limit of about 3.7 for $\gamma=B_{c 2}^{a b} / B_{c 2}^{c}$ is estimated close to $\gamma \approx 4.5$ for the first single crystal of the whole class $\left(\mathrm{NdO}_{0.82} \mathrm{~F}_{0.12} \mathrm{FeAS}[32]\right)$. The reduced $\gamma$ of these samples compared with Refs. 7, 33 might be ascribed also to disorder provided in both cases a less anisotropic Fermi surface sheet is involved like in $\mathrm{MgB}_{2}$ [34]. The $B_{c 2}(T)$ data for the $10 \% R_{N}$ criterion show no PL up to $33 \mathrm{~T}$. Hence, for $B_{c 2}^{c}$ no PL is expected. To simulate the substantially enhanced $d B_{c 2} / d T$ at $T_{c}$ which, estimated in the weak-coupling regime, is $\propto B_{c 2}(0)$ 35], we adopt using Eq. (6) $\gamma_{i m p} \approx 50 \mathrm{meV}$, i.e. a situation intermediate between clean and dirty limit. The ratio between the BCS-coherence length $\xi_{0}$ and the mean free path $l$ would be 3.6 justifying roughly the above use of the dirty limit theory valid for $\xi_{0} / l \gg 1$. The increase of $T_{c}$ is difficult to understand within a simple $s$-wave scenario. For instance, in $\mathrm{V}_{3} \mathrm{Si}, \mathrm{Nb}_{3} \mathrm{Sn}$ and $\mathrm{MgB}_{2}$ the slopes of $B_{c 2}$ raise with increasing disorder measured by $\rho_{0}$, whereas their $T_{c}$ 's slightly decrease [29, 34]. Here the raise of $T_{c}$ might result from (i) an enhanced density of states $N(0)$ due-to disorder, if the Fermi energy is located in a tail of a broadened peak of $N(E)$, (ii) spin fluctuation $s$-wave pairing supported by weak el-ph interaction being pair-breaking 
in $p$ and $d$ channels, and/or (iii) suppression of competing (local) antiferromagnetism by a reduction of the nesting. If the pairing in the clean samples is indeed unconventional, "nonmonotonous" disorder dependencies should first cause a weakening of that pairing followed by an improved $s$-wave pairing as probably observed here.

To summarize, we reported a high-field study of a $\mathrm{LaO}_{0.9} \mathrm{~F}_{0.1} \mathrm{FeAs}_{1-\delta}$ sample with improved superconductivity. It exhibits a rather high slope $d B_{c 2} /\left.d T\right|_{T_{c}} \approx-$ $5.4 \mathrm{~T} / \mathrm{K}$ to $-6.6 \mathrm{~T} / \mathrm{K}$ depending on the used definition of $B_{c 2}(T)$. In all cases at lower $T$ a flattening of the $B_{c 2}(T)$ curve points to $\mathrm{PL}$ behavior with $B_{c 2}(0) \approx 63 \mathrm{~T}$ to $68 \mathrm{~T}$ extrapolated. We ascribe this behavior to disorder effects in an extended $s$-wave state. Thus, controlled disorder provides a useful tool to study the pairing symmetry of these novel superconductors. In view of the achieved improved $d B_{c 2} /\left.d T\right|_{T_{c}}$ at a higher $T_{c}$-value, the introduction of As vacancies or of other defects opens new routes for optimizing their properties. Its study should provide also a deeper insight into the specific role of As $4 p$ orbitals played in the formation of quasiparticles relevant for the physical properties including the magnetic excitations. The PL found here suggests to continue measurements at least up to $70 \mathrm{~T}$ in order to elucidate, whether there is still much room for increasing $B_{c 2}$ beyond that range. Apparently, the solution of this problem will affect the evaluation of future high-field applications.

We thank M. Deutschmann, R. Müller, S. Pichl, R. Schönfelder, and S. Müller-Litvanyi for technical assistance . Discussions with H. Eschrig, A. Gurevich, I. Mazin, and K. Koepernik are grateful acknowledged.

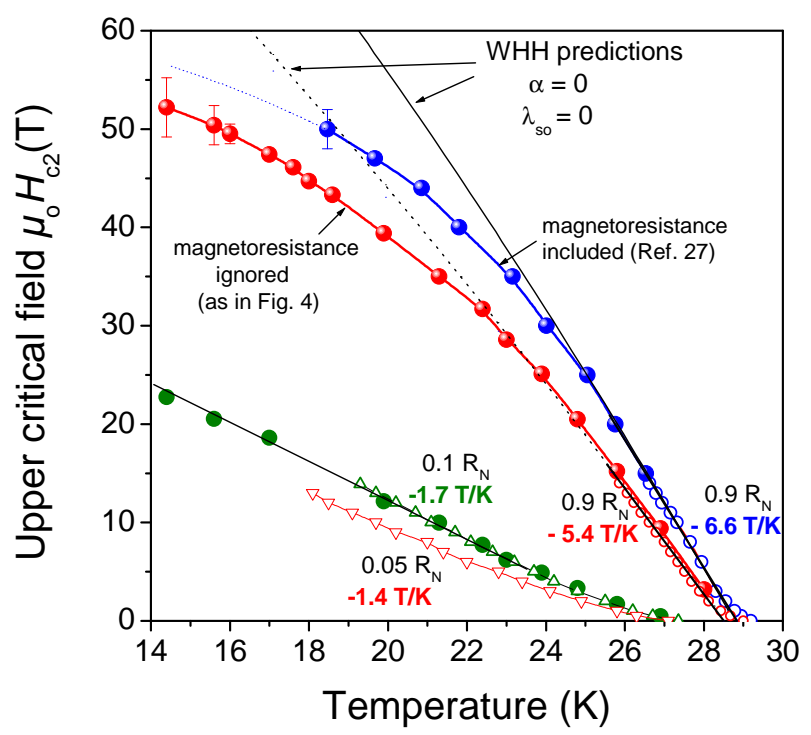

FIG. 5: (Color online) Upper critical field vs. T from 90\%, $10 \%$ and $5 \%$ of the normal state resistance $R_{N}$. Open (filled) symbols: dc field data - Fig. 2 (pulsed field data - Fig. 3).
[*] Corresponding author. drechsler@ifw-dresden.de

[1] Y. Kamihara et al., J. Am. Chem. Soc. 130, 3296 (2008).

[2] L. Boeri et al., Phys. Rev. Lett. 101 , 026403 (2008).

[3] G. Xu et al., Europhys. Lett. 8267002 (2008).

[4] I.I. Mazin et al., Phys. Rev. Lett. 101, 057003 (2008).

[5] M.M. Korshunov and I. Eremin, arXiv: 0804.1793.

[6] T.Y. Chen et al., Nature 453, 1224 (2008).

[7] A. Dubroka et al., Phys. Rev. Lett. 101, 097011 (2008).

[8] K. Haule et al., Phys. Rev. Lett. 100, 226402 (2008).

[9] K. Haule and G. Kotliar arXiv: 0805.0722.

[10] J. Dong et al., Europhys. Lett. 83, 27006 (2008).

[11] Z.-Y. Weng, arXiv: 0804.3228.

[12] D. Singh et al., Phys. Rev. Lett. 100, 237003 (2008).

[13] J.-Y. Lin et al., Phys. Rev. B 59, 6047 (1999).

[14] C. Petrovic et al., ibid. 66, 054534 (2002).

[15] A.P. Mackenzie et al., Phys. Rev. Lett. 80, 161 (1998).

[16] R.J. Radtke et al., Phys. Rev. B 48, 653 (1993).

[17] F. Hunte et al., Nature 453, 903 (2008).

[18] A. Sefat et al., Phys. Rev. B 77, 174503 (2008).

[19] G.F. Chen et al., Phys. Rev. Lett. 100, 247002 (2008).

[20] N.R. Werthamer et al., Phys. Rev. 147295 (1966).

[21] H. Luetkens et al., Phys. Rev. Lett. 101, 097009 (2008).

[22] H.-H. Klauss et al. ibid., 077005 (2008).

[23] S.-L. Drechsler et al., Phys. Rev. Lett. 101257004 (2008).

[24] H.-J. Grafe et al., Phys. Rev. Lett. 101, 047003 (2008).

[25] C. Hess et al., erXiv:0811.1601.

[26] X. Zhu et al., Supercond. Sci. Technol. 21, 105001 (2008).

[27] Within another approach, especially to have an alternative determination of $B_{c 2}$, where the magnetoresistance was taken into account, the resistance in the normal state $R_{N}(T)$ has been fitted between $T_{c}$ and $80 \mathrm{~K}$ resulting in $R_{N}(T)=7.74+1.7 \cdot 10^{-2} T^{1.4}$ given in $\mathrm{m} \Omega$ and $T$ in $\mathrm{K}$.

[28] H. Krug et al., Physica B 294-295, 605 (2001).

[29] T.P. Orlando et al., Phys. Rev. B 19, 4545 (1979).

[30] M. Schossmann and J.P. Carbotte, ibid. 39, 4210 (1989).

[31] S.V. Shulga et al., J. Low Temp. Phys. 129, 93 (2002).

[32] Y. Jia et al. Appl. Phys. Lett. 93, 032503 (2008).

[33] S. Weyeneth et al., arXiv:0806.1024.

[34] A. Gurevich, Physica C 456, 160 (2007).

[35] L.N. Bulayevskii et al., Phys. Rev. B 38, 11290 (1988). 